\title{
9
}

\section{A Study on Urban Water Reuse Management Modeling}

\author{
Changyu Zhang, Kumaraswamy Ponnambalam and Paul \\ Calamai
}

Water reuse is being recognized as a sustainable urban water management strategy and is becoming increasingly attractive in urban water resources management. This paper focuses on urban water reuse planning and management in the context of sustainable development, and introduces a state of the art urban water reuse management model which utilizes a network flow optimization model and various stochastic programming methods. The objective of the model is to minimize the overall cost of the system subject to technological, societal and environmental constraints, therefore the optimum allocation of urban water resources can be obtained. Uncertainty issues associated with water demand and treatment quality are modeled by introducing stochastic programming methods, namely, twostage stochastic recourse programming and chance-constraint programming. An application is presented in order to demonstrate the modeling process and to analyze the impact of uncertainties. This research is important in aiding the achievement in sustainable urban water resource management practices.

Zhang, C.C., K. Ponnambalam and P. Calamai. 2006. "A Study on Urban Water Reuse Management Modeling." Journal of Water Management Modeling R225-09. doi: 10.14796/JWMM.R225-09.

(C) CHI 2006 www.chijournal.org ISSN: 2292-6062 (Formerly in Intelligent Modeling of Urban Water Systems. ISBN: 0-9736716-2-9) 


\subsection{Introduction}

\subsubsection{Background and Motivation}

During the last century, rapid urbanization and population growth have resulted in many environmental problems. Among the most serious are water shortage and pollution. Human activities affect the natural water ecological cycle in many ways such as: the reduction of forested areas, the shrinkage of the grass land for grazing, and the spread of urban growth resulting in increased rainwater lost to runoff; overexploitation of groundwater resources has decreased groundwater levels and caused problems of seawater intrusion; toxic industrial discharge and the extensive use of chemical fertilizers have polluted water supply system. Many regions in the world are facing the great challenge of water shortage and pollution, and the situation is getting worse.

Many communities in the world seek a sustainable water management strategy. The objective of sustainable development of urban water systems is to satisfy the water demands at a lower affordable cost with minimum environmental and social impacts. Reuse of treated wastewater for beneficial purposes offers a potential additional water supply. General water reuse opportunities are listed in the following table.

Table 9.1 General water reuse opportunities (Asano et al., 1996).

\begin{tabular}{|c|c|}
\hline General Category & Specific Uses \\
\hline Agricultural irrigation & Crop irrigation \\
\hline Landscape irrigation & $\begin{array}{l}\text { Parks, golf courses, residential, school yard, } \\
\text { freeway medians, cemeteries, greenbelts }\end{array}$ \\
\hline Industrial reuse & $\begin{array}{l}\text { Cooling, boiler feed, processing water, } \\
\text { heavy construction }\end{array}$ \\
\hline Groundwater recharge & $\begin{array}{l}\text { Groundwater replenishment, salt water } \\
\text { intrusion, subsidence control }\end{array}$ \\
\hline $\begin{array}{l}\text { Recreational and } \\
\text { environmental reuse }\end{array}$ & $\begin{array}{l}\text { Lakes and ponds, marsh enhancement, } \\
\text { stream flow augmentation, fisheries, } \\
\text { snowmaking }\end{array}$ \\
\hline $\begin{array}{l}\text { Other non-potable urban } \\
\text { reuse }\end{array}$ & $\begin{array}{l}\text { Fire protecting, air conditioning, toilet } \\
\text { flushing }\end{array}$ \\
\hline Potable reuse & $\begin{array}{l}\text { Blending in water supply, pipe to pipe } \\
\text { water supply }\end{array}$ \\
\hline
\end{tabular}


Water reuse also reduces the rigorous and costly treatment requirements for effluent discharge to surface waters. By eliminating effluent discharges through water reuse, a community may be able to avoid or reduce the need for expanding the costly advanced wastewater treatment processes. In short, a water reuse program can serve both water conservation and pollution abatement purposes (US EPA, 1992). Therefore, water reuse is considered an important element of sustainable urban water resources management.

Due to the complex nature of the water resource problems, water reuse planning and management modeling is being investigated in order to promote such practices. Among the many management models, optimization models are very important in providing essential information for decision makers about the water resources management and planning. Meanwhile, in practice, uncertainty issues associated with reuse water demand and water quality have to be considered as they often pose a great challenge in better modeling a water reuse system.

\subsubsection{Review on Urban Water Allocation and Reuse Modeling}

It has been long recognized that integrative management modeling is essential for evaluating and optimizing treatment and reuse of wastewater. Water reuse planning and management modeling can provide a systematic approach to assessing the potential reuse water market, and identifying and evaluating water reuse opportunities among major users in the system.

Bishop and Hendricks (1971) presented one of the first models in water reuse by employing a simple un-capacitated transhipment model with consideration of treatment and transportation costs in linear form. Mulvihill and Dracup (1974) tried to minimize the cost of supplying water from several sources, including the provision for recycling reclaimed water. Another study was Pingry et al.'s (1979) nonlinear model which took into account both flow requirements and water quality. All models during this period could be regarded as simple models. During the 1980's, more complex water reclamation and reuse models were presented. Perhaps the most important was the one developed by Ocanas and Mays (1981). Their water reuse model could be used to determine the optimum reuse of wastewater on a regional basis. The model included quality constraints and provided the flexibility of adding more quality parameters. Moreover, the model took into consideration quality changes caused by users and treatment plants resulting from the inclusion of reuse water as influent. Schwartz and Mays (1984) and Vieira and Lijklema (1989) developed models using dynamic programming. Their models offered the ability to determine the 
optimal size and location of treatment plants with consideration of water demand over certain time periods.

Over the last decade, environmental and health risk issues associated with water reuse began appearing in some water reuse studies. Jacques and Anastasia (1996) discussed the risk analyses of wastewater reclamation and reuse. Their objective was to show how engineering risk analysis may be used to quantify the risk of wastewater reuse and to lead the way for developing a decision support system for wastewater reclamation and reuse. They suggested that the expected benefits and costs could be expressed as a function of risk and that Geographic Information Systems (GIS) could be used for data processing. Oron (1996) presented an integrative approach for reusing domestic treated wastewater with consideration over levels of treatment, water supply and demand, transportation and storage requirements, and environmental pollution and health risk.

Some industrial ecological studies also contributed to water reuse modeling in the last decade. Studies in industrial ecology generally focus on characterizing material and energy flows in industrial systems and analyzing cases where modifications of the material and energy flows could result in environmental and economical benefits. Keckler's material reuse model (1998) is one of the industrial ecology models demonstrating water flow design in industrial parks. The model identified water reuse opportunities between different industries using a linear programming method. Based on Keckler's material reuse model, Nobel (1998) developed an ArcView GIS model to quantitatively identify and display the results of matched water reuse source-destination pairs on GIS maps. Please refer to Zhang (2005) for more detailed discussion and applications of GIS in water reuse modeling.

In summary, studies on urban water reuse management modeling exist in the literature. However, the uncertainty issues associated with water demand and treatment quality in water reuse modeling has not received much attention. Variations in demand for reclaimed water, as well as the uncertainty in water quality are major concerns in evaluating water reuse opportunities. For example, depending on different weather condition, reuse water demands for irrigation might change dramatically. In the case of residential and municipal reuse water uses, weather conditions also play an important role in the rate of water consumption. For industry water reuse, production activity and manufacturing levels are usually affected by economics. Uncertainty with effluent quality often reflects treatment instability. Quality uncertainty has a significant effect on water reuse 
management such as safety considerations during periods of noncompliance with water quality standards.

Consideration of uncertainty issues requires incorporation of stochastic programming into the water reuse model. The next section introduces the methodologies used to develop the water reuse management model, namely, the network flow optimization model, two-stage stochastic recourse programming and chance-constrained programming.

\subsection{Methodology}

\subsubsection{The Network Flow Optimization Model}

As a special linear programming method, the minimum network flow optimization model is typically described in terms of supplies and demands for a commodity in a network system. In the model, nodes are defined as model transfer points, arcs interconnect the nodes, and commodity flows on the arcs (Ford et al., 1962). The attributes of nodes are usually supplies or demands, while the attributes of arcs are usually the flow capacities that limit the flows along them. Nodes with supplies are often referred to as sources and nodes with demands are often referred to as sinks. An optimal solution of the network flow model can be regarded as the overall least cost (or maximum value) set of flows for which supplies find their ways through the network to meet the demands.

Applying the model to our water reuse system, water users (demand sites) are treated as sinks, and water suppliers (supply sites) are treated as sources and both are nodes in the network. The links from sources to sinks are arcs (source-destination pairs). The amount of water transferred from source to sink is the flow rate, which is usually the decision variable of the optimization problem.

According to Bazaraa et al. (1977) the network optimization model can be expressed in a general form as following:

$$
\begin{array}{ll}
\text { Minimize: } & \sum_{(i, j) \in A} C_{i, j} x_{i, j} \\
\text { Subject to: } & \sum_{(j, k) \in A} x_{j, k}-\sum_{(k, i) \in A} x_{k, i}=b_{k} \quad \forall k \in N \\
& 0 \leq x_{i, j} \leq U_{i, j} \quad \forall(i, j) \in A
\end{array}
$$


where:

$$
\begin{aligned}
& i, j \in N \quad \text { nodes of the network; } \\
& (i, j) \in A \quad \text { arcs of the network; } \\
& b_{k} \text { is the net demand of node } k,\left(\text { if } b_{k} \geq 0\right) ; \\
& \left.\left|b_{\mathrm{k}}\right| \text { is the net supply of node } k \text {, (if } b_{k} \leq 0\right) ; \\
& \operatorname{Arc}(k, i) \in A \quad \operatorname{arcs} \text { leaving node } k ; \\
& \operatorname{Arc}(j, k) \in A \quad \operatorname{arcs} \text { entering node } k ; \\
& C_{i, j} \text { is the unit flow cost on } \operatorname{arc}(i, j), \\
& x_{i, j} \text { is the flow rate on arc }(i, j) ; \\
& U_{i, j} \text { is the flow capacity on } \operatorname{arc}(i, j) .
\end{aligned}
$$

A simple node-arc network diagram for the proposed water allocation and reuse model is shown in Figure 9.1, where: nodes 1 and 2 represent a surface water source and a groundwater source respectively, node 3 represents a water treatment plant; node 4 through 7 represent water users, nodes 8,9 and 10 represent a wastewater treatment plant collecting and treating wastewater with 2 levels of wastewater treatment processes generating water for reuse, and node 11 represents a receiving water body.

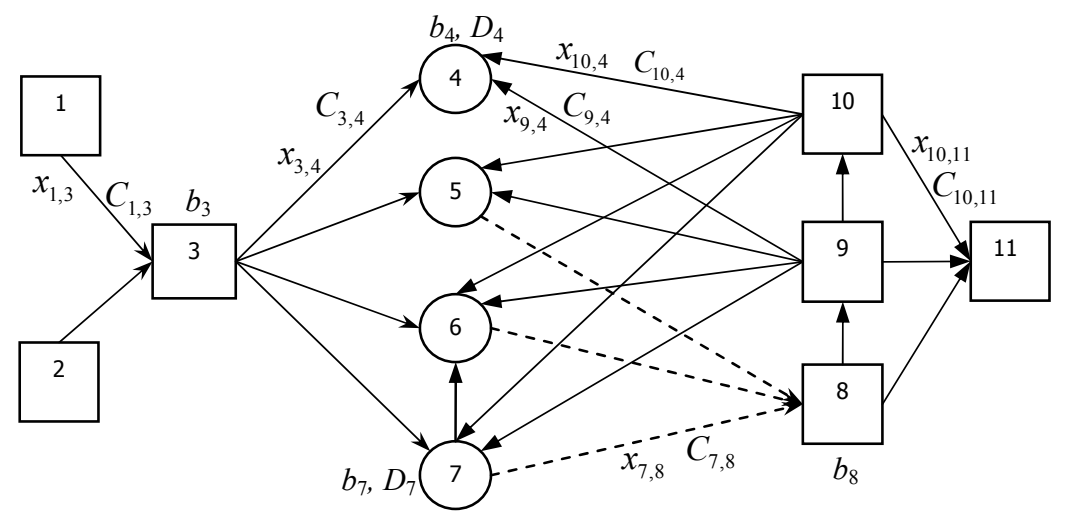

Figure 9.1 Node-arc network with variables and parameters.

Some typical model variables and parameters are also shown in the figure where $x, C, b$ are as previously defined. For water reuse modeling, demand $D$ for users has to be introduced. Demand $D$ is the total amount of water required by the user, usually consists of net demand $b$ and the amount of 
water discharged from the user after use. For irrigation users (e.g., node 4 in Figure 9.1), demand $D_{4}$ equals its net demand $b_{4}$, since all water supplied are consumed without discharge. As well, for water reuse modeling, other parameters such as water quality and facility's capacity have to be considered. Thus, in addition to flow balance constraints, water demand, water quality, and capacity limit make up the main constraints for the water reuse model.

\subsubsection{Stochastic Programming Methods}

In planning urban water resource allocation and reuse, uncertainty issues in water demand and treatment system reliability must be considered. Variations in demand for reclaimed water, as well as the uncertainty in water quality, are major concerns in evaluating water reuse opportunities. The two-stage stochastic recourse programming method and the chanceconstrained stochastic methods are two efficient stochastic programming methods for modeling these uncertainty issues.

\section{Two-Stage Stochastic Recourse Programming}

In the two-stage stochastic recourse model, first-stage decisions are decisions that are implemented before knowing the outcome of the random event. These first-stage decisions can be regarded as proactive, and are often associated with planning issues such as capacity expansion or aggregate production planning; while second-stage decisions are decisions that are implemented after knowing the outcome of the random event. Second-stage decisions can be regarded as reactive and are often associated with operating decisions. Second-stage decisions usually depend upon the first-stage decisions; therefore they are often used to model a response to the observed outcome. In general, this type of planning involves setting up responsive policies to adapt to the revealed outcome. For example, in water allocation models, the first-stage decisions correspond to water flow quantities from supply nodes to demand nodes, where demand might be modeled using random variables. When demand exceeds the amount of water supplied, policy may dictate that customer demand be backlogged at some penalty cost. The level of response (the amount backlogged) depends on the amounts supplied and demanded under uncertainty. The second stage variables and the corresponding penalty costs balance the violation caused by the outcome of the uncertain parameters. Thus, the planning 
decisions obtained would minimize the overall expected costs (or maximize the overall profits) under considerations of uncertainty with demands.

The following illustration of two-stage recourse programming is excerpted from Suvrajeet et al. (1999). For a generic two-stage formulation under recourse policy, the same notation used for deterministic linear programming (LP) can be applied. Generally, the constraints of the deterministic LP are written as:

$$
A x=b
$$

Under uncertainty, the sub-matrix $A_{1}$ (of $A$ ) and the sub-vector $b_{1}$ (of $b$ ) can be considered as rows that contain only deterministic parameters. Set $R$ represents the remaining rows in $A$ that contains at least one uncertain element. $a_{i}$ is referred to as the $i^{\text {th }}$ row vector in A, and use $\widetilde{a}$ to reflect the presence of random variables in the vector a. Let $g_{i}>0$ denote the penalty cost for violating the target $\widetilde{b}_{i} \cdot y_{i}{ }^{+}$and $y_{i}{ }^{-}$are defined as the non-negative second-stage variables representing the surplus and shortfall used for compensating the violations caused by the randomness of demand $\widetilde{b}_{i}$. When the random vectors $\left\{\widetilde{a}_{i}, \widetilde{b}_{i}\right\}_{i \in R}$ are discrete random variables, let $S_{i}$ denote an index set of all outcomes of the random vector $\left\{\widetilde{a}_{i}, \widetilde{b}_{i}\right\}_{i \in R}$ and let $p_{i s}=P\left\{\left(\widetilde{a}_{i}, \widetilde{b}_{i}\right)=\left(a_{i s}, b_{i s}\right)\right\}$ define the probability that $\left(\widetilde{a}_{i}, \widetilde{b}_{i}\right)=\left(a_{i s}, b_{i s}\right)$.

Thus, the following prototypical model allows a simple recourse policy:

$$
\begin{array}{cl}
\text { Minimize: } & c^{\prime} x+\sum_{i \in R} g_{i}\left\{\sum_{s \in S_{i}} p_{i s}\left(y_{i s}{ }^{+}+y_{i s}{ }^{-}\right)\right\} \\
\text {Subject to: } & A_{1} x=b_{1}, \quad x \geq 0 \\
& a_{i s} x-y_{i s}{ }^{+}+y_{i s}{ }^{-}=b_{i s} \\
& L_{i} \leq x \leq U_{i}
\end{array}
$$

where:

$\sum_{i \in R} g_{i}\left\{\sum_{s \in S_{i}} p_{i s}\left(y_{i s}{ }^{+}+y_{i s}^{-}\right)\right\}=$the expected recourse cost of choosing $\mathrm{x}$ in the

first stage,

$L_{i}$ and $U_{i}=$ the lower and upper bonds of $x$ respectively. 


\section{Chance-Constrained Stochastic Programming}

Chance-constrained stochastic programming is another major approach for dealing with uncertain parameters in optimization problems in which chance constraints can be used for modeling uncertainty where degrees of compliances with the constraints are specified.

In water reuse modeling, this method can be used to model the uncertainties with water quality. As discussed earlier, in the two-stage recourse model, the uncertainty is modeled by introducing penalty costs associated with the second stage variables to respond to the violations caused by the randomness. In some applications, such as production and inventory models, obtaining the penalty costs is possible. However, in many applications, such as when safety relevant restrictions like levels of a water reservoir, water quality, etc. are modeled, the penalty costs cannot be modeled practically. In such situations, chance constraints can be used to guarantee the feasibility of decisions at certain desired levels. In our water reuse model, it is more appropriate to ensure a certain probability of quality compliance. The illustration below is based on Gottfried and Weisman (1973).

In a general optimization problem with objective function $y$ and inequality constraints $g_{i}(X) \leq b_{i}$ assume that some of the technological coefficients, decision variables, or constraints include random correlations. With uncertainty in the constraints, it may be impractical to insist that $b_{i}$ exceed $g_{i}(X)$ at all times. However, by employing chance-constrained programming, such constraints can be limited to a low level of probability of violation as follows. Let $K_{i}$ be the desired minimum probability level of compliance, so that the constraints can be rewritten as:

$$
P\left\{g_{i}(X) \leq b_{i}\right\} \geq K_{i}
$$

In this way the optimization problem is to optimize (maximize or minimize) the expected value of the objective function $Z, E(Z)$ with constraints having the above form:

Optimize: $E(Z)$

Subject to: $P\left\{g_{i}(X) \leq b_{i}\right\} \geq K_{i}, \quad i=1,2, \ldots, m$.

Using the mean and standard deviation of $g_{i}(X)$ to approximate $g_{i}(X)$ : 


$$
g_{i}(X) \cong \mu_{g_{i}(X)}+t_{i} \sigma_{g_{i}(X)}
$$

where $t_{i}$ is the number of standard deviations from the mean, often called the standard normal variate. Since

$$
\mu_{g_{i}(X)}=E\left(g_{i}(X)\right)
$$

The certainty equivalent of Equation 9.11 becomes

$$
E\left(g_{i}(X)\right)+t_{i} \sigma_{g_{i}(X)} \leq b_{i}
$$

So the deterministic equivalent optimization problem becomes:

Optimize: $E(Z)$

Subject to: $E\left(g_{i}(X)\right)+t_{i} \sigma_{g_{i}(X)} \leq b_{i} \quad t=1,2, \ldots, m$

Applied to our study, the uncertainty issue with water quality is modeled with the chance-constrained stochastic programming method.

In the case where the coefficients of the objective function $(Z)$ are uncertain, the uncertainty can be modeled using the mean-variance method developed by Markowitz (1992). The objective function is:

$$
\text { Optimize: } \quad E(Z) \pm \theta \operatorname{Var}(Z)
$$

where $\operatorname{Var}(Z)$ is the variance of the objective function $(Z)$ and $\theta$ is the risk aversion parameter; "+" for minimization and "_" for maximization. The risk aversion parameter indicates the willingness of paying money (or not to receive a high return) to reduce risk or avoid uncertainty in investment or planning (Baker, 2001). Applying to our water reuse modeling study, mathematically, the uncertainty with water price or water treatment cost can be modeled with this method since they are the coefficients of the total cost function, i.e., the objective function.

\subsection{Formulation of the Water Reuse Management Model}

As discussed earlier, the optimization model was developed based on the network flow optimization model. The basic constraints of this model are adapted and revised from the model developed by Ocanas and Mays (1981). 
As discussed in the previous section, two-stage stochastic recourse programming and chance-constrained programming methods are introduced into the model's constraints and objective function to allow for uncertain demands and water quality modeling. The model's variables, constraints and objective function are specified in what follows.

\subsubsection{Model Decision Variables}

Decision variables include all flow rates between source nodes and sink nodes in the network system. They are defined as follows:
$X W T_{l, j} \quad$ flow rate of treated water from water treatment plant
node $l$ to user node $j$;
$X W W T_{h, j}$ flow rate of treated water from wastewater treatment
plant node $h$ to user node $j$;
$X W R_{j, r}$ flow rate of wastewater from node $j$ to receiving water $r$;
$X T W W T_{j, l}$ flow rate of wastewater from node $j$ to WWTP node $l$;
$X S T_{i, l} \quad$ flow rate of fresh water from source $i$ to WTP $l$;
$X G T_{k, l} \quad$ flow rate from groundwater source $k$ to WTP $l$;
$X W W T R_{h, r}$ flow rate of wastewater from WWTP $h$ to receiver $r$;
$X_{t, j} \quad$ flow rate of water from user $t$ to user $j$ for reuse;
$E X S_{j} \quad$ flow rate of external water supply to user $j$.

The units for flow rates are in $\mathrm{m}^{3} /$ day. The decision variables in this network flow model are the flow rate on each arc of the system.

\subsubsection{Model Parameters and Required Input}

For urban water reuse planning and management, the parameters and input are listed below:
US set of users;
$S U$ set of surface water sources;
$G R \quad$ set of groundwater sources;
$R \quad$ set of receiving water bodies;
$P \quad$ set of pollutants;
WTP set of water treatment plants;
WWTP set of wastewater treatment plants; 
$D E M_{j}$ water demand of user $j$;

$N D L_{j} \quad$ net demand of user $j$;

$L T_{l} \quad$ water losses at water or wastewater treatment plant $l$;

$Q R\left(P_{n}\right)_{r}$ maximum mass discharge of pollutant $n$ acceptable by receiving water body $r$;

$C S\left(P_{n}\right)_{i}$ pollutant $n$ concentration of surface water source $i$;

$C G\left(P_{n}\right)_{k}$ pollutant $n$ concentration of groundwater source $k$;

$\operatorname{STD}\left(P_{n}\right)_{j}$ maximum pollutant $n$ concentration required by user $j$;

$C W T\left(P_{n}\right)_{l}$ pollutant $n$ concentration leaving water treatment plant $l$;

$C W W T\left(P_{n}\right)_{h}$ pollutant $n$ concentration leaving wastewater treatment plant $h$;

$C\left(P_{n}\right)_{t} \quad$ pollutant $n$ concentration leaving water user $t$;

$C A P_{l}$ and $C A P_{h}$ capacities of WTP $l$ and WWTP $h$ respectively.

The units for pollutant concentrations are $m g / l$ and the units of the mass discharge of pollutants are $m^{3} \cdot m g / l$, and demand and capacity are in $m^{3} /$ day.

\subsubsection{Model Constraints}

\section{Demand constraints}

The deterministic demand constraints which force the model to satisfy the demand for all users are given as:

$$
\sum_{l \in W T P} X W T_{l, j}+\sum_{h \in W W T P} X W W T_{h, j}+\sum_{t \in U S} X_{t, j}+E X S_{j} \geq D E M_{j} \quad \forall j \in U S
$$

To ensure that these constraints are satisfied in a situation of water shortage in the system an external supply source is added. When the user demands $D E M j$ are random, applying two-stage stochastic recourse programming, the stochastic form of these demand constraints becomes:

$$
\sum_{l \in W T P} X W T_{l, j}+\sum_{h \in W W T P} X W W T_{h, j}+\sum_{t \in U S} X_{t, j}+E X S_{j}-S P_{s, j}+S F_{s, j} \geq D E M_{j} \quad \forall j \in U S, s \in S
$$

where $S P_{s, j}$ and $S F_{s, j}$ are non-negative second stage variables denoting the surplus and the shortfall of case $s$ (of the scenario set $S$ ) to the demand respectively. 
The corresponding total penalty cost which will augment the objective function is:

$$
C_{\text {penalty }}=\sum_{j \in U S} C P_{j}\left\{\sum_{s \in S} p_{s, j}\left(S P_{s, j}+S F_{s, j}\right)\right\}
$$

where $C P_{j}$ denotes the unit penalty cost, $p_{s, j}$ denotes the probability of case $s$ in the scenario set $S$.

According to probability theory, the scenarios can be generated once the characteristic of the distribution of the random variable (which is the demand in this case) becomes known.

\section{Network flow balance constraints}

These constraints ensure that the flows are balanced at each node in the network. For users these constraints are:

$$
\begin{aligned}
& \sum_{l \in W T P} X W T_{l, j}+\sum_{h \in W W T P} X W W T_{h, j}+\sum_{t \in U S} X_{t, j}+E X S_{j}-\sum_{r \in R} X W R_{j, r}-\sum_{l \in W W T} X T W W T_{, l}-\sum_{t \in U S} X_{j, t} \\
& =N D L_{j} \forall j \in U S
\end{aligned}
$$

Capacity constraints

For water treatment plants these constraints are given by

$$
\sum_{i \in S U} X S T_{i, l}+\sum_{k \in G R} X G T_{k, l} \leq C A P_{l} \quad l \in W T P
$$

\section{Quality requirement constraints}

These constraints force the flows in the system to satisfy the quality requirements of each user (i.e., that the pollutant concentrations are less than specified criteria). These constraints are derived from the assumption that the concentration of the water being delivered to the user is derived from the mixture of all flows entering that user node. Therefore the constraint requires that the resulting concentration of pollutants in the mixture be no more than the user's upper-bound. 
The deterministic form of these constraints is:

$$
\begin{aligned}
& {\left[\sum_{i \in S U} C S\left(P_{n}\right)_{i} X S_{i, j}+\sum_{k \in G R} C G\left(P_{n}\right)_{k} X G_{k, j}+\sum_{l \in W T P} C W T\left(P_{n}\right)_{l} X W T_{l, j}+\right.} \\
& \left.\sum_{h \in W W T P} C W W T\left(P_{n}\right)_{h} X W W T_{h, j}+\sum_{t \in U S} C\left(P_{n}\right)_{t} X_{t, j}\right]-S T D\left(P_{n}\right)_{j}\left[\sum_{i \in S U} X S_{i, j}+\right. \\
& \left.\sum_{k \in G R} X G_{k, j}+\sum_{l \in W T P} X W T_{l, j}+\sum_{h \in W W T P} X W W T_{h, j}+\sum_{t \in U S} X_{t, j}\right] \leq 0 \quad \forall j, n
\end{aligned}
$$

Uncertain coefficients of water quality may include $C S\left(P_{n}\right)_{i}, C G\left(P_{n}\right)_{k}$, $C W W T\left(P_{n}\right)_{h}, C W W T\left(P_{n}\right)_{h}$, and $C\left(P_{n}\right)_{t}$. For simplicity denote the left hand side of the equation (9.23) by $g_{j}(X)$. According to the chance-constrained stochastic programming, and assuming a desired quality compliance level of $K_{j}$ and the corresponding risk factor $t_{j}$, the stochastic form of these quality constraints are:

$$
E\left(g_{j}(X)\right)+t_{j} \sigma_{g_{j}(X)} \leq 0 \quad \forall j, n
$$

As an important stochastic programming method, chance-constrained programming offers a comprehensive strategy in modeling quality compliance in water reuse system based on various risk levels.

Other constraints such as water availability constraints and the constraints in modeling the leveled wastewater reclamation processes are discussed in Zhang (2005).

\subsubsection{Objective Function}

The objective of this water reuse optimization model is to determine the minimum cost solution to the problem of supplying water to every user in the system when water reuse is included. These costs generally include the water and wastewater treatment costs, the transportation costs, the infrastructural costs and penalty costs for modeling the uncertainty as discussed earlier. The objective of this model can be written as:

$$
\begin{aligned}
& \text { Minimize: } \\
& C(X)=C_{(W T P)}+C_{(W W T P)}+C_{(\text {Transportation })}+C_{(\text {(nffrastructure })}+C_{(\text {penalty })}
\end{aligned}
$$

The model results are the optimum allocation of water in the system, that is, the flow rates between each supply site and demand site. The optimization 
model ensures that the cost is minimized and all the constraints are satisfied. Therefore, water reuse potentials between reuse water supply sites and demand sites can be further analyzed.

\subsection{Model Implementation and Discussion}

\subsubsection{Example Problem and Input Data}

The model was implemented to a hypothetical problem as illustrated in Figure 9.2 below. The feasible water supply and demand connections are to be determined by the optimization model, so the connections shown in the figure are just possible ones.

The system includes one water treatment plant (WTP), one wastewater treatment plant, one receiving water body and fifteen users. Two dummy sources were assumed. The wastewater treatment plant is assumed to employ three levels of wastewater treatment processes with the basic level of treatment satisfying the wastewater discharge requirements and the other two levels of treatments generating reclaimed water for reuse (WWTP1, WWTP2 and WWTP3). The major water users include ten ICI (Industrial, Commercial and Institutional) water users, three major residential areas (RE) and two golf courses (GF) as irrigation water users.

Connection rules used in the network are described as following: a WTP can supply any users; the dummy source can supply any users; some ICI users can supply other users; ICI users and Residential users discharge to WWTP1; Golf courses' irrigation has no discharge; WWTP1 only discharges to WWTP2 and Receiving water body; WWTP2 can supply WWTP3, all users and the Receiving water body; WWTP3 can supply all users and discharge to the Receiving water body. These rules were realized by defining an appropriate node-arc incidence matrix in the network flow optimization model.

For water quality requirements, four common water quality parameters were considered here as examples, namely Total Suspended Solids (TSS), Biochemical Oxygen Demand (BOD), Chemical Oxygen Demand (COD), and Total Organic Carbon (TOC). Some input data used are also listed in the following table. The parameters used here are assumed to be independent and the correlation between them can be modeled using the method of correlation coefficient which is introduced later in this paper. Other quality parameters could be added. 

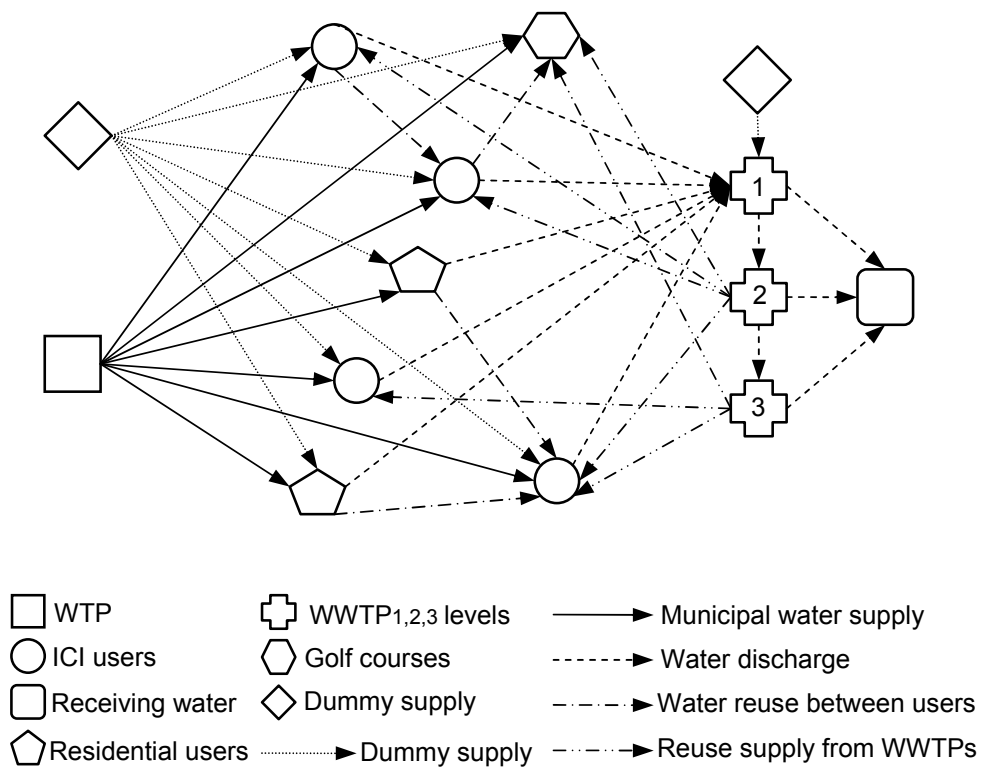

Figure 9.2 Network diagram of the case study system.

Table 9.2 Water demand and quality data for the water users.

\begin{tabular}{c|c|cccc|cccc}
\hline \multirow{2}{*}{$\begin{array}{c}\text { User } \\
\text { Index }\end{array}$} & Demand* & \multicolumn{4}{|c|}{ Influent (mg/l) } & \multicolumn{4}{c}{ Effluent (mg/l) } \\
\cline { 3 - 9 }$\left(\mathrm{kgpd}^{* *}\right)$ & TOC & TSS & BOD & COD & TOC & TSS & BOD & COD \\
\hline ICI-1 & 424 & 50 & 100 & 20 & 20 & 137 & 220 & 180 & 150 \\
ICI-2 & 127 & 50 & 100 & 20 & 20 & 240 & 147 & 100 & 250 \\
ICI-3 & 122 & 50 & 100 & 30 & 30 & 160 & 106 & 100 & 140 \\
ICI-10\# & 134 & 80 & 180 & 60 & 75 & 3869 & 257 & 600 & 1000 \\
RE-1 & 688 & 25 & 50 & 40 & 50 & 290 & 350 & 200 & 400 \\
RE-2 & 825 & 25 & 50 & 40 & 50 & 160 & 220 & 300 & 500 \\
RE-3 & 1155 & 25 & 50 & 40 & 50 & 160 & 220 & 400 & 600 \\
GF-1 & 260 & 80 & 200 & 150 & 250 & N/A & N/A & N/A & N/A \\
GF-2 & 130 & 80 & 200 & 150 & 250 & N/A & N/A & N/A & N/A \\
\hline
\end{tabular}

\subsubsection{Results and Discussion}

Firstly, as an urban water allocation and reuse management model, it is capable of obtaining the optimal allocation of urban water resources with consideration of water reuse in the given system. By considering water 
reuse, the water users' demands are supplied by various water sources including municipal water supply and reuse water supplies. All these water resources are allocated to users under the condition that the overall costs of the system are minimized and water demands and quality requirements are satisfied. Secondly, by incorporating stochastic modeling methods, the model can help decision makers in making the best decisions regarding the uncertainty issues associated with water reuse planning and management. In this paper, the discussions are focused on results considering uncertainties.

\section{Uncertainty with Demand}

Water demands of five users (RE-1, RE-2, RE-3, GF-1 and GF-2) are considered uncertain and their mean values are assumed known. The Coefficient of Variation $(\mathrm{CV})$ is used to measure the levels of variation of these demands. $\mathrm{CV}$ is defined as:

$$
C V=\frac{\text { Standard Deviation }}{\text { Expected Value(Mean })}=\frac{\sigma}{\mu}
$$

For simplicity, a normal distribution was assumed for the uncertain variables in this research. In order to differentiate the levels of variability in demands, a set of $C V$ was used, i.e., $0.05,0.10,0.20$ and 0.50 , corresponding to variability levels from low to high. The probability distribution corresponding to the demand of user RE-1 under this set of $C V$ is shown in Figure 9.3.

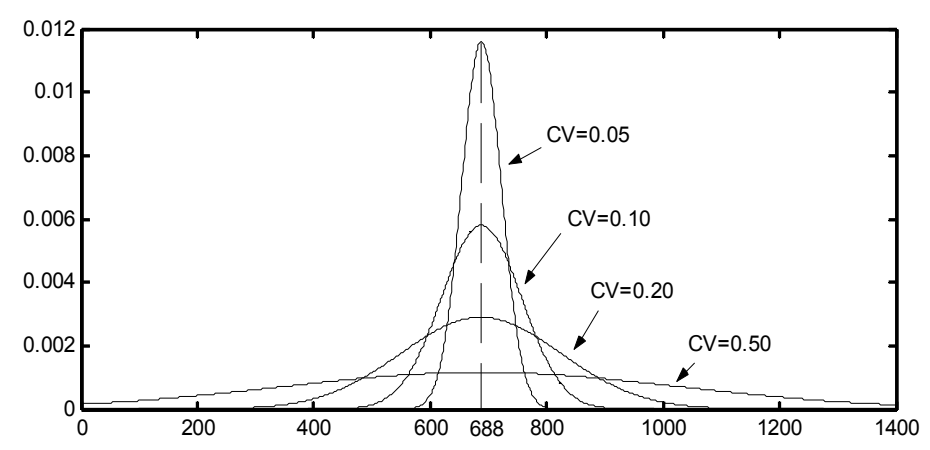

Figure 9.3 Distributions under different CVs for user RE-1. 
For water demand modeling, the relationships between demands among the users in the system can be considered by introducing the concept of correlation coefficient. For example, under similar weather conditions, the irrigation water demand of one golf course would reflect the demand of other golf courses, but this relationship would not hold between a golf course and an industry. Mathematically, these kinds of relationship between variables can be modeled by their correlation coefficients, a measure that determines the degree to which two variable's movements are associated. The correlation coefficient is often denoted by $r$ and is defined as:

$$
r_{i, j}=\frac{\operatorname{Cov}\left(x_{i}, x_{j}\right)}{\sigma_{x_{i}} \sigma_{x_{j}}}
$$

where:

$$
\begin{aligned}
r_{i, j} & =\text { correction coefficient between variable } x_{i} \text { and } x_{j} ; \\
\operatorname{Cov}\left(x_{i}, x_{j}\right) & =\text { covariance of these two variables. }
\end{aligned}
$$

Based on the covariance of the uncertain demands, a large number of scenarios can be generated to model the random variables, in this case, the uncertain water demands. The scenario generation process was done using MATLAB function "mvnrnd" with the mean, covariance, and number of scenarios as input parameters. These scenarios are used in two-stage recourse stochastic programming to model the demand uncertainty.

By minimizing expected costs an optimal water allocation policy can be reached for which the risk of demand noncompliance is minimized. In this paper, only results with WWTP supplies are presented for the discussions. Figure 9.4 shows the $\mathrm{WWTP}_{2}$ (basic reclaimed) reuse water supplies under different levels of demand variation, that is, coefficients of variation: 0.05 , $0.10,0.20$ and 0.50 . For the users with uncertain demands (RE-1, RE-2, RE3, GF-1 and GF-2), their supplies tend to decrease as the level of demand variation gets higher. Meanwhile, the supplies for users with certain demands (ICI-1 through ICI-10) were not affected.

As we know, the smaller the coefficient of variation, the closer the uncertain variable behaves like a deterministic one. Figure 9.5 gives the objective function values, which are the costs to the overall water reuse system, optimized under cases with different demand $C V$ s. By examining this figure, we can see the trade-off between cost and demand variation: the increase of the level of demand variation costs more to the system. 


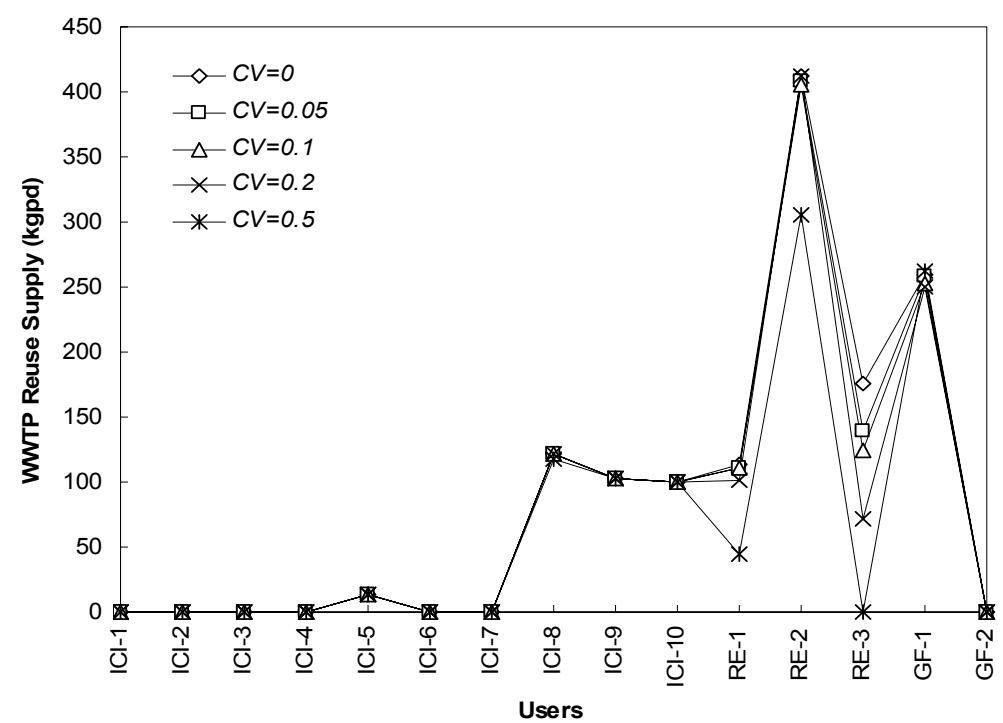

Figure 9.4 WWTP reuse supply with different coefficients of variation of demand.

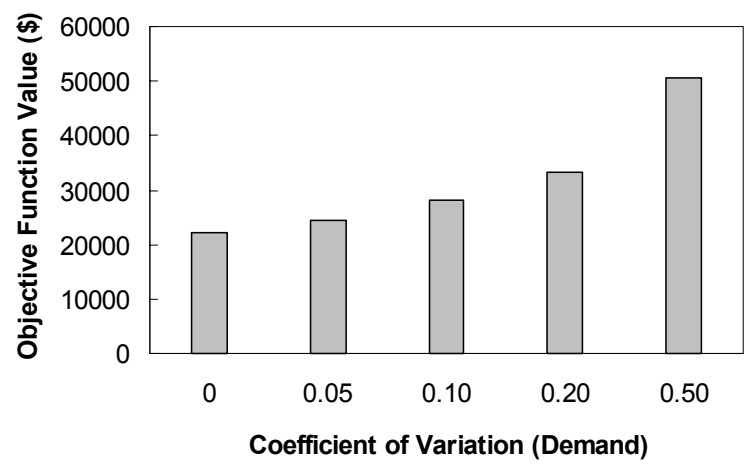

Figure 9.5 Cost-variation trade-off. 


\section{Uncertainty with Quality}

Uncertainty with effluent quality from reuse water supply sources has been modeled using the chance-constrained programming method. For modeling quality uncertainty in this study, the same set of coefficients of variation with quality is used as was used for demand uncertainty modeling. For implementing the chance-constrained programming method, the risk factors are set to $1.75,1.28,0.85$ and -0.25 , corresponding to probability levels, denoted by $p$, of quality compliance of $96 \%, 90 \%, 80 \%$ and $40 \%$ respectively. For simplicity, the quality of the WWTP basic reclaimed reuse water supply is considered uncertain. Figure 9.6 gives the results of WWTP reuse water supplies respectively under the different quality chanceconstraints with $C V=0.05$. This figure illustrates that the higher quality chance-constraints require that less water be supplied from the WWTP than WTP, which provides water with higher quality. As well, if we compare the results obtained when levels of quality variability are varied (using the coefficients of variation) under the same quality chance-constraint, the relationship between supplies and quality variability can be observed.

From these observations, we can conclude that the reliability of water quality provided by reuse water sources determines the feasibility of their use for water reuse applications. Figure 9.7 illustrates the trade-off of cost with various chance-constraints and different levels of quality variability.

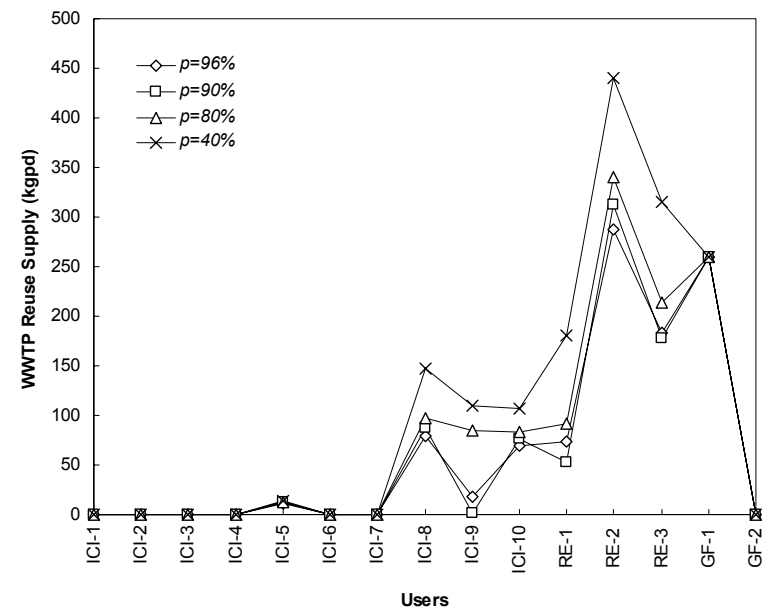

Figure 9.6 WWTP reuse water supplies under different chanceconstraints with quality variability $C V=0.05$. 


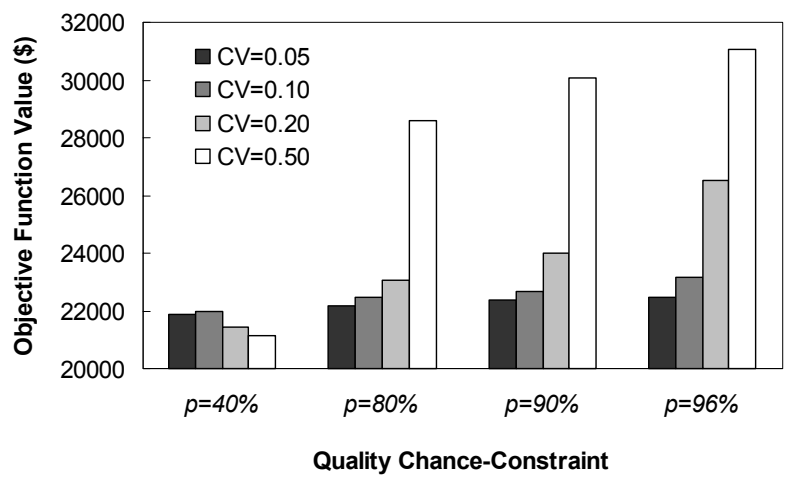

Figure 9.7 Trade-off of cost and quality chance-constraint and levels of quality variation $(\mathrm{CV}=0.05,0.10,0.20$ and 0.50$)$.

These plots show that with the same level of quality variation (quality $C V s$ ), the higher quality chance-constraint $(p)$ will require a higher cost. However a more important observation is that when the level of quality variation gets sufficiently high, the cost to the system climbs dramatically if we want to achieve the same quality chance-constraint. The conclusion that can therefore be drawn is that for a water reuse system, the most significant aspect of cost effectiveness is the quality variability of the reuse water supplies or, in other words, the reliability of water treatment for reuse.

\section{Uncertainties with Demand and Quality}

Since demand and quality uncertainties typically occur at the same time, we now look at some cases where both uncertainties occur simultaneously. Table 9.3 lists the 4 cases that were investigated and Figure 9.8 shows the WWTP reuse water supplied under these cases. Figure 9.9 illustrates the corresponding costs for these four cases.

Table 9.3 Case specifications.

\begin{tabular}{ccc}
\hline Cases & Demand Uncertainty & Quality Uncertainty \\
\hline I & deterministic & deterministic \\
II & deterministic & $C V=0.05$, chance-constraint $=96 \%$ \\
III & $C V=0.05$ & deterministic \\
IV & $C V=0.05$ & $C V=0.05$, chance-constraint $=96 \%$ \\
\hline
\end{tabular}




\section{Uncertainty with Price or Cost}

In addition to modeling of uncertainties with demand and quality, this model is also capable of modeling uncertainty with water price or water treatment cost. Here, the modeling results from a simple case of uncertainty with the WWTP $_{2}$ reuse water treatment cost is presented with the assumption of normal distribution and the mean value of $\$ 1.00$. In this case the cost risk aversion parameters $(\theta)$ were chosen to be $5 \mathrm{e}-10,0.05$ and 0.50 respectively. Figure 9.10 shows the comparison of the total costs for these risk aversion parameters based on the same condition of demand and quality uncertainties (demand $C V=0.05$, quality $C V=0.05$ and chance-constraint $p=96 \%$ ). From these results, the trade-off between risk aversion parameters and the total costs can be easily observed.

\section{Sensitivity Analysis to Price Parameters}

Besides the analyses discussed above, several experimental trials were performed to investigate the sensitivity of the proposed model to some input parameters. From the sensitivity analyses show that the WTP water price has a major effect on water reuse, namely a higher WTP water price will definitely promote water reuse and that reducing WWTP treatment costs has a positive effect on encouraging water reuse.

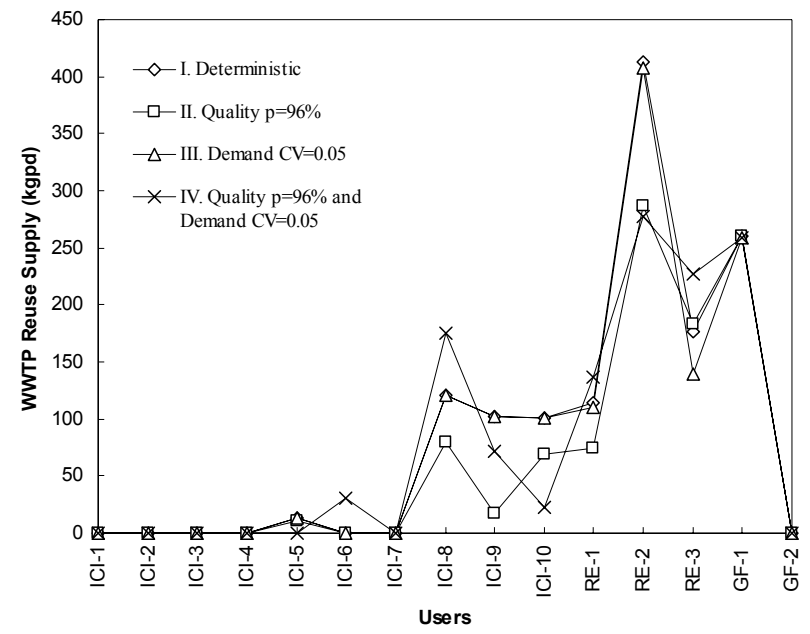

Figure 9.8 WWTP reuse water supplies under different cases of uncertainty with quality and demand. 


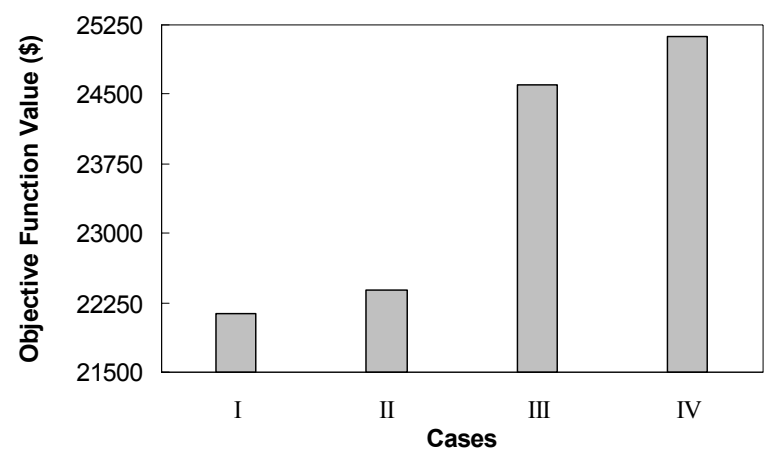

Figure 9.9 Cost for the four cases.

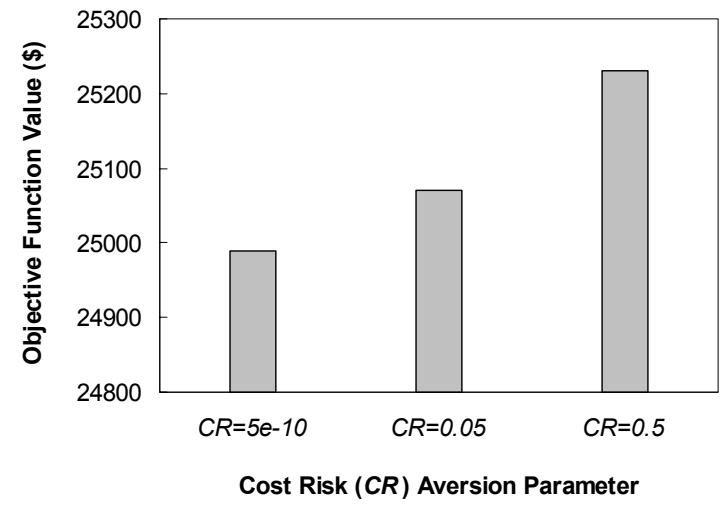

Figure 9.10 Trade-off between cost risk and total cost.

\subsection{Conclusions}

This research work investigated water reuse management modeling in the context of sustainable development. Prior to this study, uncertainty issues in water reuse modeling had not been fully addressed in the literature, so the focus of this study was stochastic modeling in water reuse systems.

An urban water allocation and reuse management model, which is capable of identifying and evaluating water reuse opportunities and 
analyzing the impact of uncertainties with water demand and quality, was developed. Specifically, the network flow optimization model, two-stage stochastic recourse programming and chance-constrained programming methods were integrated to form the basis of the model. The modeling results exemplified the trade-off between expected costs to the system and the variations with demand and quality. By examining the optimal allocation of municipal and reuse water supplies and the overall costs to the entire system, one can conclude that the stability of reuse water treatment and quality has an important impact both on the reliability of water quality compliance and on the cost effectiveness of the entire water reuse system. The model is useful in evaluating water reuse management alternatives and deriving more robust decisions in regard to uncertain demand and quality.

For the complete study introduced in this paper, please refer to Zhang (2005).

\section{References}

Asano, T., and A. Levine, "Wastewater Reclamation, Recycling and Reuse: Past, Present and Future" Water Science and Technology, Vol.33 (10-11) pp.1-14 (1996)

Baker S. L. "Economics Interactive Tutorial", Department of Health Administration, University of South Carolina. http://hadm.sph.sc.edu/ (2001)

Bazaraa M. S., John J. Jarvis and Hanif D. Sherali. "Linear Programming and Network Flows". John Wiley \& Sons, pp.420. ISBN: 0-471-63681-9 (1977)

Bennett, D. A. A Framework for the Integration of Geographical Information Systems and Modelbase Management.' International J. of GIS, Vol. 11 (4) pp.337-357 (1997)

Bishop, A and D. Hendricks, "Water Reuse Systems Analysis", Journal of the Sanitary Engineering Division (ASCE) Vol. 97 (1) pp.41-57 (1971)

Environmental Systems Research Institute (ESRI), "Understanding GIS: The Arc/Info Method". Redlands, California (1997)

Ford, L. R Jr. and D. R. Fulkerson, "Flows in Networks". Princeton University Press, Princeton, New Jersey (1962)

Gottfried B. S. and J. Weisman. "Introduction to optimization theory". Prentice-Hall, Inc., Englewood Cliffs, New Jersey (1973)

Jacques G., and P. Anastasia, "Risk Analysis of Wastewater Reclamation and Reuse". Water Science and Technology. Vol.33 (10-11) pp.297-302 (1996)

Keckler, S., "A Materials Reuse Model", Thesis for a Master of Science in Engineering, The University of Texas at Austin, Austin, Texas (1997)

Markowitz, H. M., "Portfolio Selection: Efficient Diversification of Investments". Second Edition, Cambridge, Mass., B. Blackwell Publishing (1992)

Mulvihill M. E. and J. A. Dracup, "Optimal Timing and Sizing of a Conjunctive Urban Water Supply and Wastewater System with Nonlinear Programming”, Water Resour. Res., Vol. 10 (2) pp.171-175 (1974) 
Nobel C. E., D. T. Allen, "Using Geographic Information Systems (GIS) in Industrial Water Reuse Modelling”. Trans IChemE, Vol 78 (B) (2000)

Ocanas, G. and L. Mays, “A model for Water Reuse Planning”. Water Resources Research Vol. 17 (1) pp.25-32 (1981)

Oron G., "Management Modeling of Integrative Wastewater Treatment and Reuse Systems". Water science and technology, Vol.33 (10-11) pp.95-105 (1996)

Pingry, D. W., and T. L. Shaftel, "Integrated Water Management with Reuse: A Programming Approach”. Water Resources Research Vol. 15 (1) pp.8-14 (1979)

Schwartz, M. and L. Mays, "Models for Water Reuse and Wastewater Planning”. Journal of Environmental Engineering (ASCE) Vol.109 (5) pp.1128-1147 (1983)

Suvrajeet S., and J. L. Higle, "An Introductory Tutorial on Stochastic Linear Programming Models". The University of Arizona, Tucson, Arizona (1999)

US EPA and US Agency for International Development, "Guidelines for Water Reuse". EPA/625/R-92/004; US EPA Technology Transfer, Cincinnati, Ohio (1992)

Vieira, J. and L. Lijklema. "Development and Application of a Model for Regional Water Quality Management”. Water Resources Vol. 23 (6) pp.767-777 (1989)

Zhang, Changyu. "A Study on Urban Water Reuse Management Modeling. Master of Applied Science in Eng'rg Thesis, U of Waterloo, Waterloo, ON, Canada (2005). 
